\title{
SCN1A Gene
}

National Cancer Institute

\section{Source}

National Cancer Institute. SCN1A Gene. NCI Thesaurus. Code C102447.

This gene is involved in sodium transport. 